\title{
Primary Splenic Peripheral Nerve Sheath Tumour in a Dog
}

\author{
W. Bergmann ${ }^{\star}$, I. A. Burgener ${ }^{\dagger}$, P. Roccabianca ${ }^{\ddagger}$, U. Rytz ${ }^{\dagger}$ and M. Welle* \\ * Institute of Animal Pathology and ${ }^{\dagger}$ Department of Clinical Veterinary Medicine, Vetsuisse Faculty, University of Berne, \\ Berne, Switzerland and ${ }^{\ddagger}$ Dipartimento di Patologia Animale Igiene e Sanita’ Pubblica Veterinaria-Sezione Anatomia \\ Patologica Veterinaria e Patologia Aviare, Facoltà di Medicina Veterinaria, Università di Padova, Italy
}

\begin{abstract}
Summary
An 8-year-old crossbred dog was presented with a one-month history of progressive weakness, respiratory impairment and abdominal distension. Surgical exploration revealed the presence of a splenic mass that infiltrated the mesentery and was adherent to the stomach and pancreas. The mass was composed of highly cellular areas of spindle-shaped cells arranged in interlacing bundles, streams, whorls and storiform patterns (Antoni A pattern) and less cellular areas with more loosely arranged spindle to oval cells (Antoni B pattern). The majority of neoplastic cells expressed vimentin, S-100 and glial fibrillary acidic protein (GFAP), but did not express desmin, $\boldsymbol{\alpha}$-smooth muscle actin or factor VIII. These morphological and immunohistochemical findings characterized the lesion as a malignant peripheral nerve sheath tumour (PNST). Primary splenic PNST has not been documented previously in the dog.
\end{abstract}

(C) 2009 Elsevier Ltd. All rights reserved.

Keywords: dog; peripheral nerve sheath tumour; spleen

Peripheral nerve sheath tumours (PNSTs) represent a heterogeneous group of lesions classified into benign schwannoma, benign neurofibroma, malignant schwannoma and neurofibrosarcoma (Hendrick et al., 1998; Koestner et al., 1999). Schwannomas originate from Schwann cells only, whereas neurofibromas and neurofibrosarcomas are mixed tumours composed of Schwann cells and perineural cells (Hendrick et al., 1998; Goldschmidt and Hendrick, 2002). Some authors, however, prefer to distinguish only between benign and malignant PNSTs (Hendrick et al., 1998; Goldschmidt and Hendrick, 2002) since differential diagnosis based upon morphology alone is difficult.

PNSTs are uncommon in animals and occur mostly in dogs, cattle and cats (Summers et al., 1995; Hendrick et al., 1998; Goldschmidt and Hendrick, 2002). Benign PNSTs (BPNSTs) of the nervous system are rare in dogs and cats and are found incidentally in cattle (Summers et al., 1995; Koestner et al., 1999). Malignant PNSTs (MPNSTs) of the nervous system are most commonly seen in dogs (Koestner et al., 1999).

Benign PNSTs of the skin are uncommon in cats and rare in dogs (Hendrick et al., 1998). In cattle, multiple benign cutaneous PNSTs may arise within the subcutis and the nerve plexuses (Hendrick et al., 1998; Goldschmidt and Hendrick, 2002). Malignant cutaneous PNSTs are uncommon in cats and extremely rare in large animals (Goldschmidt and Hendrick, 2002). The true incidence of canine dermal malignant PNST is difficult to estimate since confirmation of the diagnosis requires immunohistochemical examination that was not performed routinely in the past.

Although rare, PNSTs arising within the thoracic cavity and abdomen have been described in man (Bui et al., 2004; Khan et al., 2006; Rahman et al., 2006; Vyas et al., 2006; Tashiro et al., 2007), horses (Pascoe, 1982; Andreasen et al., 1993; Kirchhof et al., 1996; Stoica et al., 2001; Quinn et al., 2005), dogs (Stoica et al., 2001; Essman et al., 2002; Aupperle et al., 2007; Patterson et al., 2008; Sugiyama et al., 2008), cats (Stoica et al., 2001) and cattle (Stoica et al., 2001). Herein we report the clinical, 
microscopical and immunohistochemical findings related to the occurrence of a PNST within the spleen of a dog. To the best of our knowledge, primary splenic PNSTs have not been described previously in man or animals.

An 8-year-old, neutered female, crossbred dog was presented with a one month history of progressive weakness, respiratory impairment and abdominal distension. Clinical examination revealed loss of general body condition, mild hypothermia (body temperature $37.6^{\circ} \mathrm{C}$ ), low heart rate $(64$ beats $/ \mathrm{min})$ and a generalized mild increase in lung sounds on inspiration. The abdomen was distended, with palpable enlargement of the spleen. Haematological and serum biochemical examinations revealed the presence of a stress leucogram, thrombocytosis and mild hypoglycaemia. The urinary sediment was unremarkable. Ultrasonography confirmed the presence of a large splenic mass, with suspected rupture but minimal peritoneal effusion. There were no lesions suggestive of metastasis on thoracic radiography. On laparotomy, the mass was seen to infiltrate the mesentery and was attached to the fundic area of the gastric wall and to the distal left limb of the pancreas (Fig. 1). Aberrant vessels had formed between the splenic mass and the caudal pole of the left kidney. The tumour was dissected from the surrounding tissue and the aberrant vascular trunk ligated. After partial gastrectomy and pancreatectomy, the tumour was completely excised and had a weight of $600 \mathrm{~g}$. Follow-

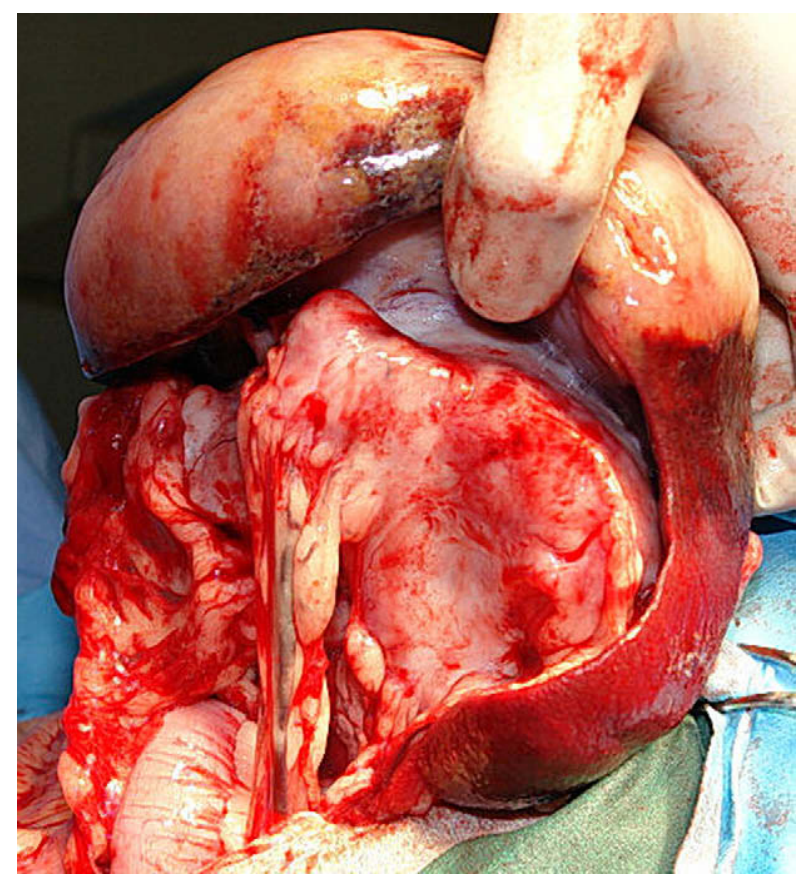

Fig. 1. Splenic tumour during surgery. The mass infiltrates the mesentery and is attached to the stomach and the pancreas. ing discharge, the dog remained well for three months, but thereafter started to vomit. An ultrasound examination at this time revealed the presence of masses within the liver and mesentery. The dog was humanely destroyed without permission for necropsy examination.

The excised spleen contained a non-encapsulated, multilobulated, infiltrative, marbled mass $(5 \times 7.5$ $\times 11 \mathrm{~cm}$ ) of soft consistency. Representative samples taken from the lesion were fixed in 10\% neutral buffered formalin, processed routinely and sections were stained with haematoxylin and eosin (HE). Serial sections were subject to immunohistochemical examination with the following primary antibodies (all from Dako, Glostrup, Denmark unless otherwise stated): rabbit polyclonal anti-factor VIII ( 1 in 400), rabbit polyclonal anti-S-100 ( 1 in 400; Zymed, San Francisco, USA), mouse monoclonal anti-Desmin ( 1 in 200; Novocastra Ltd, Newcastle UK), mouse anti- $\alpha$ smooth muscle actin (1 in 2000), mouse anti-vimentin ( 1 in 20,000; Sigma, St Louis, USA), mouse anti-glial fibrillary acidic protein (GFAP; 1 in 3000) and rabbit polyclonal anti-human c-kit (1 in 200). Secondary detection was performed with an avidin-biotin-peroxidase kit according to the manufacturer's instructions (Vector laboratories, Burlingame, CA, USA).

Microscopically, the mass was non-encapsulated, poorly demarcated and replaced the majority of the splenic parenchyma. Neoplastic tissue infiltrated the surrounding adipose tissue and mesentery. The mass had a biphasic growth pattern. Some regions were highly cellular and composed of interlacing bundles and streams of spindle-shaped cells growing in a storiform pattern or as whorls around blood vessels and nerves. These cells had poorly defined eosinophilic fibrillar cytoplasm and spindle-shaped basophilic nuclei and were associated with variable amounts of collagenous stroma (Antoni A pattern; Fig. 2). Other areas were less cellular and composed of loosely arranged spindle to oval cells with inconspicuous cytoplasm and a small hyperchromatic round nucleus surrounded by a copious myxoid, often microcystic, matrix (Antoni B pattern). In these areas numerous lymphatic vessels were markedly dilated (Fig. 3). The mitotic rate in both areas was less than one per $\times 400$ high-power field. Immunohistochemical labelling revealed that almost $100 \%$ of the neoplastic cells expressed cytoplasmic vimentin, S-100 and GFAP (Fig. 4), but there was no expression of desmin, $\alpha$ smooth muscle actin, factor VIII or c-kit. These findings supported a diagnosis of a primary splenic malignant PNST.

On the basis of morphological appearance it is difficult to differentiate PNST from other mesenchymal tumours, particularly those of perivascular origin 


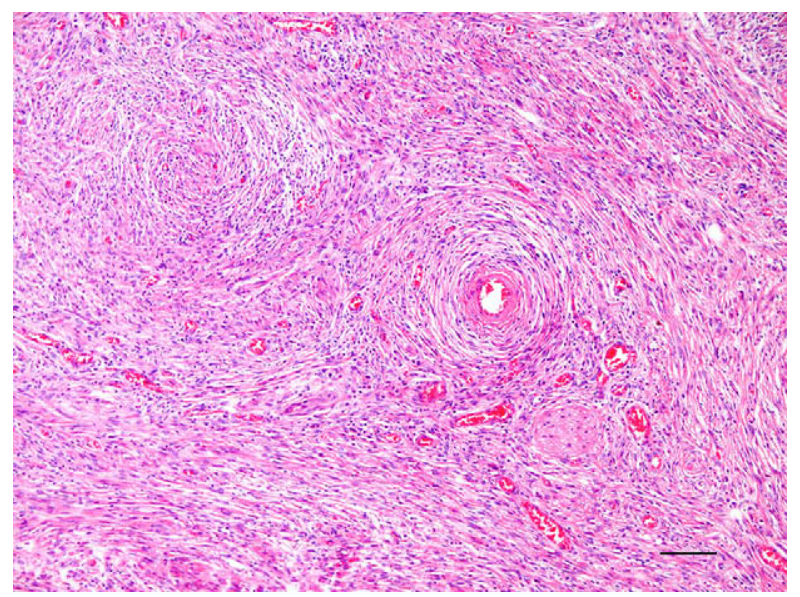

Fig. 2. Highly cellular area composed of interlacing bundles, streams and whorls of spindle-shaped cells (Antoni A pattern). HE. Bar, $50 \mu \mathrm{m}$.

(Stoica et al., 2001; Goldschmidt and Hendrick, 2002). Definitive diagnosis requires immunohistochemistry (IHC), electron microscopy or genetic analysis (Stoica et al., 2001). No specific immunohistochemical markers are able to define a PNST, so a panel of reagents is generally employed. PNSTs are, like all spindle cell tumours, positive for vimentin, indicating a mesenchymal origin (Pérez et al., 1996; Chijiwa et al., 2004). S-100 identifies Schwann cells and consequently most PNSTs express this molecule (Koestner et al., 1999; Chijiwa et al., 2004). There may be variable expression of GFAP, nerve growth factor receptor (NGFR) and neuron specific enolase (NSE) (Koestner and Higgins, 2002; Chijiwa et al., 2004). However, other tumours may also express the combination of S-100, GFAP, NGFR and NSE (Chijiwa et al., 2004). The inclusion of antibodies specific

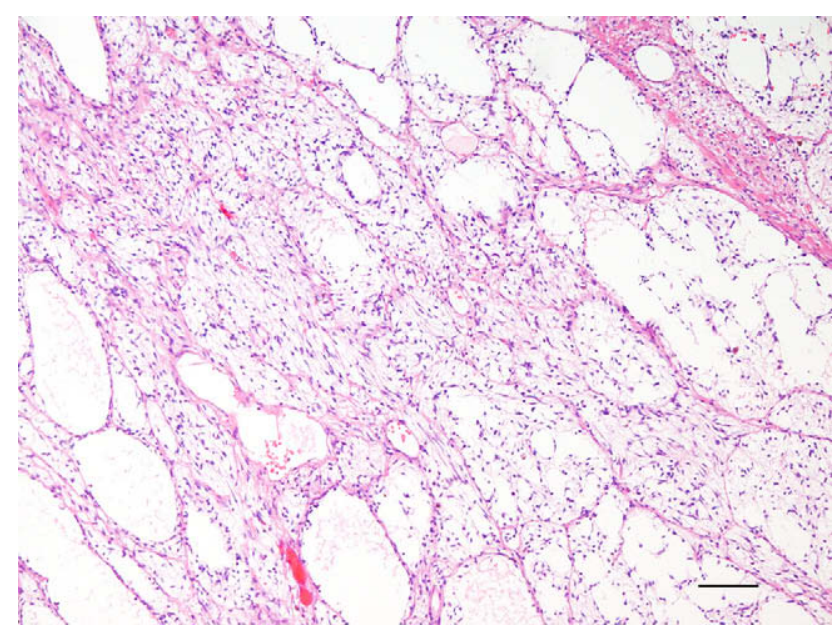

Fig. 3. Area of less cellular, more loosely arranged spindle cells, surrounded by a copious myxoid matrix (Antoni B pattern). HE. Bar, $50 \mu \mathrm{m}$.

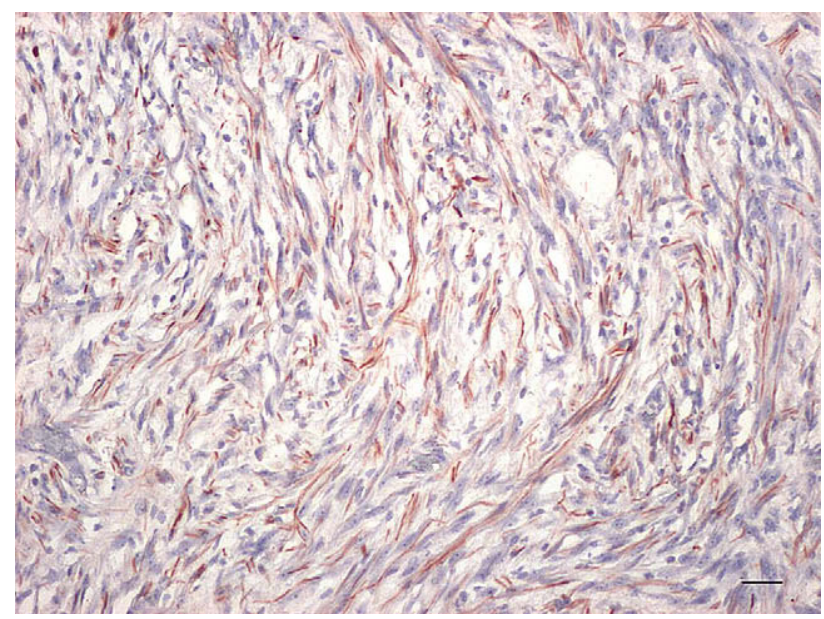

Fig. 4. Neoplastic spindle cells with cytoplasmic expression of GFAP. IHC. Bar, $50 \mu \mathrm{m}$.

for factor VIII, desmin and $\boldsymbol{\alpha}$-smooth muscle actin in the diagnostic panel can assist in distinguishing tumours of vascular or myofibroblastic origin. Use of immunolabelling for c-kit can exclude the possibility of gastrointestinal stromal tumour (GIST). In the present case, confirmation of the identity of this tumour was only made possible following evaluation of the lesion immunohistochemically with such a panel of antisera.

\section{Acknowledgment}

The authors thank Dr. Chiara Brachelente for performing the c-kit immunohistochemistry.

\section{References}

Andreasen CB, Hedstrom OR, Alison P (1993) Mediastinal schwannoma in a horse: cytologic, histologic, and immunochemical evaluation. Veterinary Clinical Pathology, 22, 54-59.

Aupperle H, März I, Ellenberger C, Buschatz S, Reischauer A et al. (2007) Primary and secondary heart tumours in dogs and cats. Fournal of Comparative Pathology, 136, 18-26.

Bui TD, Nguyen T, Huerta S, Gu M, Hsiang D (2004) Pancreatic schwannoma. A case report and review of the literature. Fournal of the Pancreas, 10, 520-526.

Chijiwa K, Uchida K, Tateyama S (2004) Immunohistochemical evaluation of canine peripheral nerve sheath tumors and other soft tissue sarcomas. Veterinary Pathology, 41, 307-318.

Essman SC, Hoover JP, Bahr RJ, Ritchey JW, Watson C (2002) An intrathoracic malignant peripheral nerve sheath tumor in a dog. Veterinary Radiology and Ultrasound, 43, 255-259.

Goldschmidt MH, Hendrick MJ (2002) Benign peripheral nerve sheath tumor (neurofibroma, schwannoma). In: 
Tumors in Domestic Animals, 4th Edit., DJ Meuten, Ed., Iowa State University Press, Ames, pp. 95-96.

Hendrick MJ, Mahaffey EA, Moore FM, Vos JH, Walder EJ (1998). In: WHO Histological Classification of Mesenchymal Tumors of Skin and Soft Tissue of Domestic Animals, Vol. II, FY Schulman, Ed., Armed Forces Institute of Pathology, Washington, DC, pp. 25-27.

Khan AA, Schizas AM, Cresswell AB, Khan MK, Khawaja HAT (2006) Digestive tract schwannoma. Digestive Surgery, 23, 265-269.

Kirchhof N, Scheidemann W, Baumgärtner W (1996) Multiple peripheral nerve sheath tumors in the small intestine of a horse. Veterinary Pathology, 33, 727-730.

Koestner A, Bilzer T, Fatzer R, Schulman FY, Summers BA et al. (1999). In: Histological Classification of Tumors of the Nervous System of Domestic Animals, Vol. 5, FY Schulman, Ed., Armed Forces Institute of Pathology, Washington, DC, pp. 26-27.

Koestner A, Higgins RJ (2002) Primary tumors of the peripheral nervous system. In: Tumors in Domestic Animals, 4th Edit., DJ Meuten, Ed., Iowa State University Press, Ames, pp. 731-735.

Pascoe PJ (1982) Colic in a mare caused by a colonic neurofibroma. Canadian Veterinary fournal, 23, 24-27.

Patterson CG, Perry RL, Steficek B (2008) Malignant peripheral nerve sheath tumor of the diaphragm in a dog. Fournal of the American Animal Hospital Association, 44, 36-40.

Pérez J, Bautista MJ, Rollón E, de Lara FC, Carrasco L et al. (1996) Immunohistochemical characterization of hemangiopericytomas and other spindle cell tumors in the dog. Veterinary Pathology, 33, 391-397.
Quinn GC, Fews D, Scase TJ, Pearson GR (2005) Malignant peripheral nerve sheath tumor of the heart in a horse. Veterinary Record, 157, 847-849.

Rahman M, Cook DS, Ellis G, O'Keefe PA (2006) Malignant peripheral nerve sheath tumor of the heart. Asian Cardiovascular and Thoracic Annals, 14, 425-427.

Stoica G, Tasca SI, Kim HT (2001) Point mutation of neu oncogene in animal peripheral nerve sheath tumors. Veterinary Pathology, 38, 679-688.

Sugiyama A, Morita T, Shimada A, Tsuka T, Okamoto Y et al. (2008) Primary malignant peripheral nerve sheath tumor with eosinophilic cytoplasmic globules arising from the greater omentum in a dog. Fournal of Veterinary Medical Science, 70, 739-742.

Summers BA, Cummings JF, deLahunta A (1995) Neoplasia and the peripheral nervous system. In: Veterinary Neuropathology, L Duncan, Ed., Mosby-Year Book, St Louis, pp. $472-481$.

Tashiro T, Kawakita C, Takai C, Yoshida T, Sakiyama S et al. (2007) Primary pulmonary malignant peripheral nerve sheath tumor: a case report. Acta Cytologica, 51, 820-824.

Vyas FL, Jesudason MR, Samuel R, Govil S, Jesudason SR (2006) Schwannoma of bile duct: a case report. Tropical Gastroenterology, 27, 50-51.

$\left[\begin{array}{l}\text { Received, October 29th, } 2008 \\ \text { Accepted, March 30th, } 2009\end{array}\right]$ 